\title{
Palatal Schwannoma: An Analysis of 45 Literature Reports and of an Illustrative Case
}

\author{
Vivek Dokania1® Anagha Rajguru ${ }^{1}$ Vishwakarma Mayashankar ${ }^{2} \quad$ Indranil Mukherjee ${ }^{3}$ \\ Bhagyashree Jaipuria ${ }^{4}$ Devika Shere ${ }^{5}$ \\ ${ }^{1}$ Department of Ear, Nose, and Throat, Krishna Institute of Medical \\ Sciences Deemed University, Karad, Maharashtra, India \\ ${ }^{2}$ Department of Ear, Nose, and Throat, HBT Medical College and Dr RN \\ Cooper Municipal General Hospital, Juhu, Mumbai, Maharashtra, India \\ ${ }^{3}$ Department of Ear, Nose, and Throat, Gouri Devi Institute of Medical \\ Sciences and Hospital, Rajbandh, Durgapur, West Bengal, India \\ ${ }^{4}$ Department of Ear, Nose, and Throat, Lokmanya Tilak Municipal \\ Medical College and Hospital, Sion, Mumbai, Maharashtra, India \\ ${ }^{5}$ Department of Ear, Nose, and Throat, Rajiv Gandhi Medical College, \\ Thane, Maharashtra, India \\ Int Arch Otorhinolaryngol 2019;23:e360-e370. \\ Address for correspondence Vivek Dokania, MBBS, MS, Department \\ of Ear, Nose, and Throat, Krishna Institute of Medical Sciences, \\ Deemed University, Karad 415110, Maharashtra, India \\ (e-mail: drvivekdokania@gmail.com).
}

\begin{abstract}
Keywords

- neurilemmoma

- palate

- schwannoma

- S100

Introduction Schwannomas are benign tumors originating from differentiated Schwann cells. Being the least common intraoral neoplasm of neural origin, it is rarely seen in the palate. The literature lacks an extensive review of intraoral schwannoma confined to the palate.

Objective To review previously reported cases of palatal schwannoma along with an illustrative case, and to provide a better insight regarding clinicopathological and radiological features of this neural tumor in a rare intraoral site.

Data Synthesis We present a case of palatal schwannoma in a 16-year-old female. An additional 45 cases were identified in 2 medical database searches (PubMed and Google Scholar) published from the year 1985 onwards, and from 13 countries, in the 5 continents. The ages of the patients ranged from 3 to 84 years old. Palatal schwannoma showed a slight predilection to females, with a male/female ratio of $\sim 1: 1.81$. Hard palate involvement is almost twice greater than soft palate involvement. Surgical excision was employed in almost all of the cases, and recurrence was reported only once.

Conclusion Palatal schwannomas, although rare, have been reported both over the hard and the soft palate. They mostly present as a painless, firm, well-encapsulated, slow-growing solitary lesion over the lateral palatal aspect.

Imaging can add to suspicion and can delineate a differential diagnosis, but the diagnosis is confirmed by pathological examination. Fine-needle aspiration cytology (FNAC) is almost always inconclusive. Immunohistochemistry can assist in confirming a diagnosis, but is more important to rule out close differentials. Complete surgical excision is the treatment of choice, and recurrence or malignant transformation are extremely rare.
\end{abstract}

(D)Vivek Dokania's ORCID is https://orcid.org/0000-0002-6970-

321X.

received

March 22, 2019

accepted

April 13, 2019
DOI https://doi.org/

10.1055/s-0039-1692635. ISSN 1809-9777.
Copyright $\odot 2019$ by Thieme Revinter

Publicações Ltda, Rio de Janeiro, Brazil
License terms

c) $(1) \$$ 


\section{Introduction}

Schwannoma or neurilemmoma is a benign tumor of neuroectodermal origin that is derived from Schwann cells of the neural sheath. ${ }^{1-3}$ In 1910, Verocay first described the microscopic features of this tumor under the term neurinoma. ${ }^{4}$ The term schwannoma was introduced by Masson in $1932 .{ }^{5}$ Later, in 1935, Stout $^{3}$ used the term, neurilemmoma, and further detailed its histopathology. In 1940, Tarlov described the tumor to be of fibroblastic origin and coined the term perineural fibroblastoma. ${ }^{6}$ About between 25 and $45 \%$ of all schwannomas are found in the head and neck region, and only between 1 and $12 \%$ of them have an intraoral origin. ${ }^{7-9}$ However, the palatal location is rare. The present article presents a specific systematic review of the published literature on palatal schwannomas, along with an illustrative case.

\section{Review of the Literature}

\section{Case Presentation}

A 16-year-old female presented with a complaint of a painless swelling over the palatal region. She first noticed a small nodule 2 months before, which was gradually increasing in size. She was otherwise healthy and did not report a history of alcohol consumption or of smoking. No genetic or syndromic abnormalities were reported from her family. Her laboratory reports were unremarkable. In the intraoral examination, a solitary, nontender, firm swelling, $2.5 \times 2 \mathrm{~cm}$ in dimension, was noted over the left soft palate. The tumor had a whitish-yellow appearance, and the overlying mucosa was ulcerated ( $\mathbf{- F i g . ~ 1 ) . ~ A ~ c o m p u t e d ~ t o m o - ~}$ graphy (CT) scan revealed a well-defined, hypodense, soft tissue lesion measuring $27.8 \times 21.6 \times 18.2 \mathrm{~mm}$ involving the left side of the soft palate (-Fig. 2). With a probable clinical diagnosis of benign salivary gland tumor, the lesion

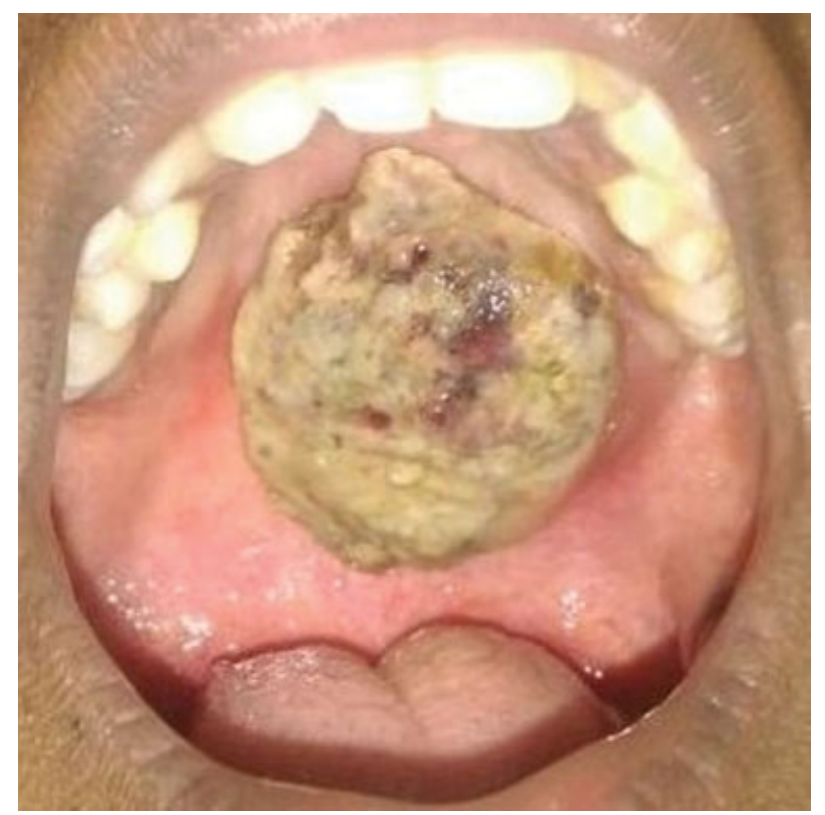

Fig. 1 Painless swelling over the left soft palate with ulceration of the overlying mucosa.

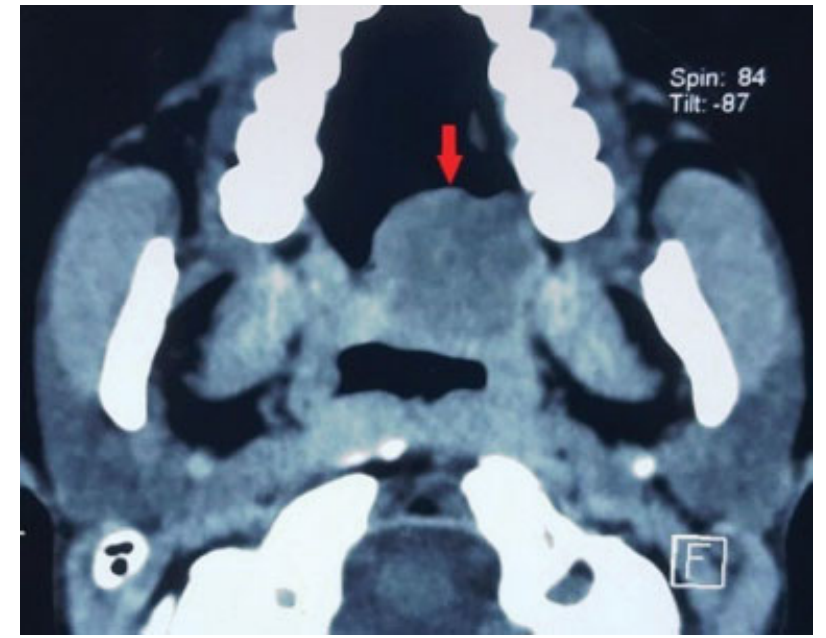

Fig. 2 Computed tomography shows a hypodense, soft tissue lesion involving the soft palate on left side (red arrow).

was completely excised and the defect was allowed to heal by secondary intention. The histological examination of the lesion revealed a predominant presence of Antoni A areas with spindle-shaped cells arranged in a palisading pattern and central acellular areas representing Verocay bodies (-Figs. 3 and 4). Some areas also showed a hypocellular and less organized arrangement, as seen in the Antoni B type. The immunohistochemical (IHC) examination with S-100 protein revealed intense positivity in the cells of the tumor (-Fig. 5). The tumor cells also showed positive expression of SRY-related HMG-box 10 (SOX-10) protein (-Fig. 6). Based on the clinical behavior, as well as on the histological and IHC findings, the final diagnosis was of a benign schwannoma of the soft palate (conventional variant).

\section{Methodology}

A systematic review of the literature was performed in August 2018 on 2 different databases (PubMed and Google Scholar). The database was searched for full-length articles and abstracts using the following Medical Subject Headings (MeSH): palate, AND schwannoma, AND/OR neurilemoma, AND/OR neurilemmoma AND hard AND/OR soft palate, AND/

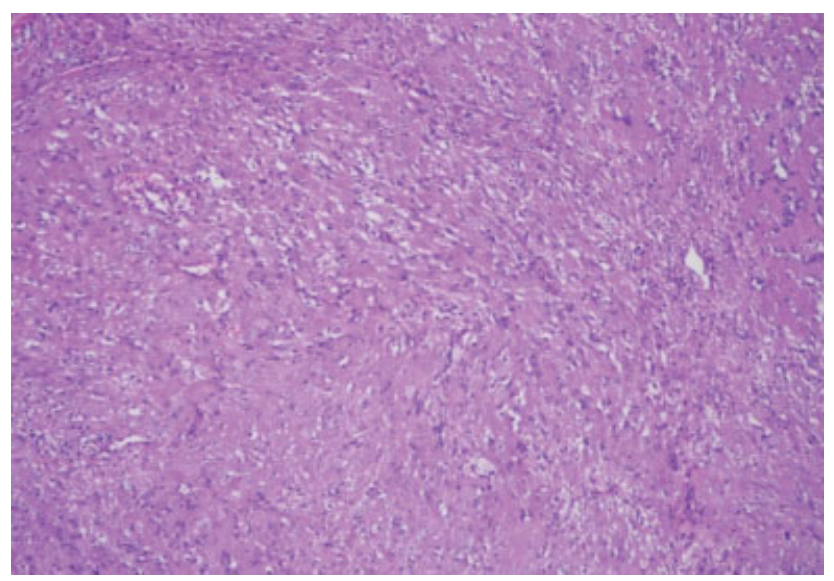

Fig. 3 Section showing a spindle cell tumor and areas of collagenization (Hematoxylin and eosin staining; 100x). 


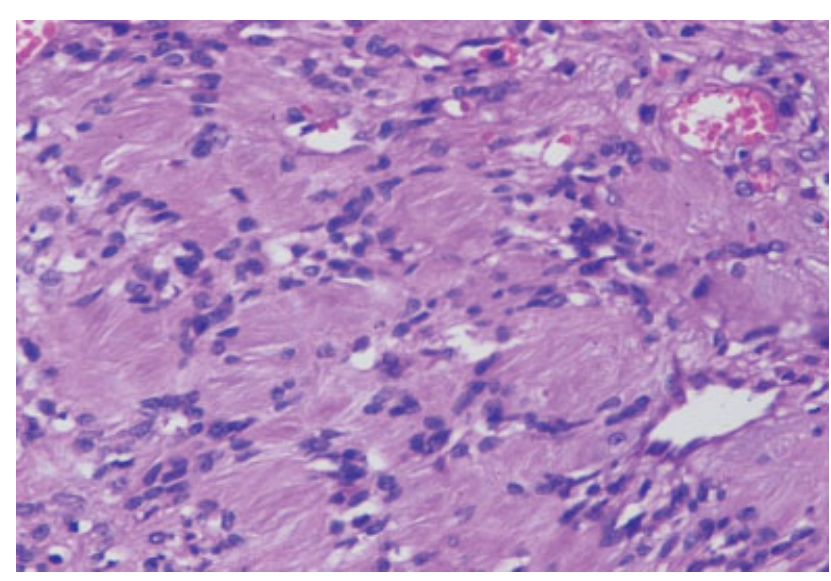

Fig. 4 Section showing proliferating fusiform cells arranged in palisading pattern and areas of acellular eosinophilic regions representing Verocay bodies (Hematoxylin and eosin staining; 200x).

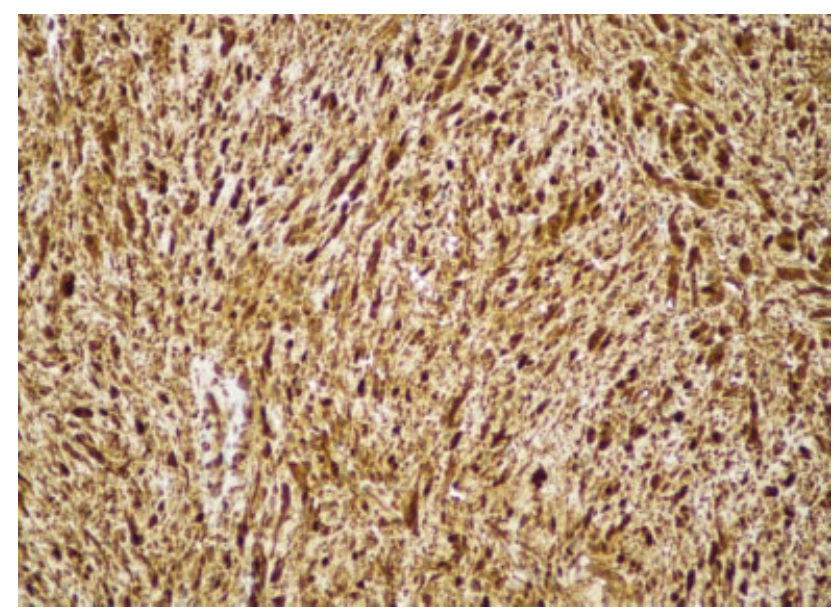

Fig. 5 Section showing tumor cells expressing strong nuclear and cytoplasmic S-100.

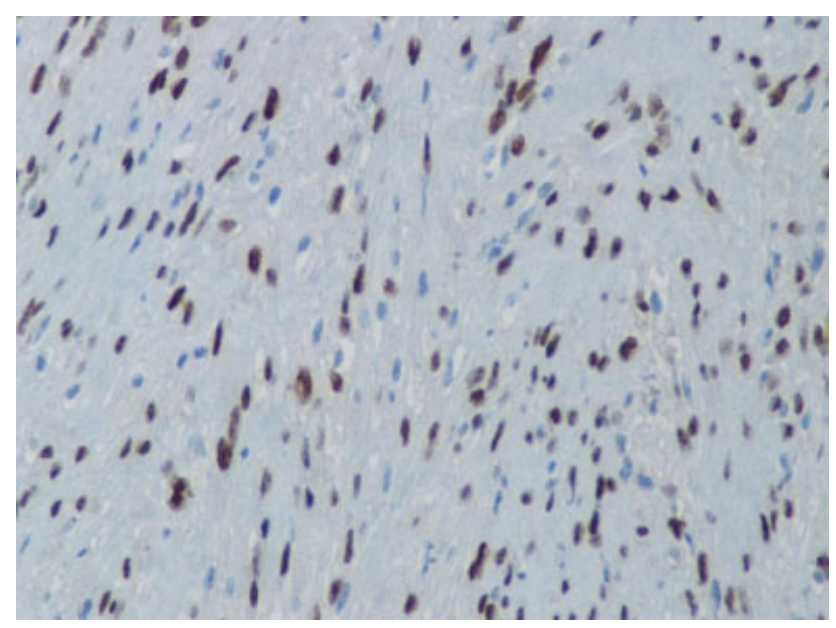

Fig. 6 Section showing tumor cells expressing SRY-related HMG-box 10 (SOX-10).

OR intraoral MINUS tongue, vestibule and other intraoral anatomical locations. The search included synonymous terms and was confined to studies or reports in humans. The review included isolated case reports or articles with up to 2 cases of palatal schwannomas published after 1984 in English, German or Japanese. Articles containing $>2$ cases of palatal schwannoma, or larger case series, were not included. Cases diagnosed as malignant schwannoma at the initial presentation were not included. No age limits were applied. Information from the included articles was collected in a predesigned Microsoft Excel (Microsoft Corporation, Redmond, WA, USA) spreadsheet.

\section{Result}

A total of 46 cases ( 45 published cases and an illustrative case) of palatal schwannoma were included in the present review. From the included articles, clinical, histopathological, radiological, and treatment findings were charted (-Tables $\mathbf{1}$ and $\mathbf{2}){ }^{10-54}$

Out of 46 compiled cases, 29 were female (64\%), and 16 were male (in 1 case, no gender was reported). ${ }^{45}$ The ages ranged from 3 to 84 years old, with an average of 30.04 years old. The mean duration of the lesion from 38 reported cases was of 25.63 months (range: 5days-20 years), while in the remaining 8 cases no information about the duration of the tumor could be retrieved (either the exact numerical duration was not stated, or the lesion was incidentally detected). ${ }^{10,17,33,38,39,44,46,47}$ Some articles reported the duration of onset in days, weeks or years. Therefore, the approximate lesional age was converted and charted into months. The incorporated cases were reported from 13 countries: India $(n=15)$, USA $(n=5)$, Brazil $(n=5)$, Japan $(n=4)$, Spain $(n=3)$, Turkey $(n=3)$, UK $(n=2)$, Germany $(n=2)$, Iran $(n=2)$, Italy $(n=2)$, Greece $(n=1)$, Morocco $(n=1)$, and Egypt $(n=1)(-$ Fig. 7$)$. The majority of the cases has been reported from India, indicating either a high prevalence or a greater awareness about the disease in that country.

The tumor involved the soft and the hard palate in 15 (32.6\%) and in 31 (67.4\%) subjects, respectively. Among the 15 soft palatal lesions, 8 involved the right side, 5 involved the left side, 1 was in the midline, and no specific site over the soft palate was mentioned in 1 case. $^{22}$ Out of 31 hard palatal lesions, 13 were confined to the right hard palate, 11 involved the left side, 5 were in the midline, 1 involved the entire hard palate, and no specific hard palatal location was reported in 1 case. $^{29}$

Most of the articles mentioned the width and length of the lesion, but the depth dimension is rarely reported. The largest diameter/dimensions of the tumor ranged from $5 \mathrm{~cm}$ to $1 \mathrm{~cm}$, with an average of $2.4 \mathrm{~cm}$ (no information about the dimension of the lesion was reported in 2 cases). ${ }^{13,17}$ When studying for an association between the duration of the lesion and lesion size, we found a weak positive correlation $(r=0.25)$. However, the correlation is statistically insignificant $(p=0.13)$ ( - Fig. 8).

Symptoms were commented in 41 cases (89\%). The remaining 5 cases were incidentally detected or asymptomatic. Painless swelling/nodule was the most common symptom, present in 40 cases (87\%). One case reported delayed pain over the tumor ${ }^{37}$ while in another patient, 


\begin{tabular}{|c|c|c|c|c|c|c|c|c|c|c|c|c|c|c|c|c|c|c|c|c|c|c|c|c|}
\hline $\begin{array}{l}\frac{\overrightarrow{5}}{5} \\
\text { 帝 } \\
>\end{array}$ & 3 & Z & i & $\frac{x}{a}$ & Z & Z & 3 & I & נ & Z & 3 & \begin{tabular}{|l}
$\frac{E}{3}$ \\
3 \\
$3 \frac{1}{2}$
\end{tabular} & Z & i & Z & $\frac{x}{a}$ & U & Z & i & $z$ & $\frac{x}{a}$ & נ & 2 & $\begin{array}{l}\vec{v} \\
\stackrel{\vec{v}}{\vec{v}} \\
\vec{v}\end{array}$ \\
\hline$\Sigma$ & ○ & 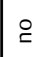 & 잉 & ஜ & 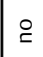 & 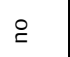 & 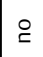 & 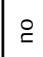 & 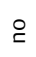 & $\stackrel{\circ}{ }$ & 잉 & 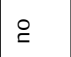 & 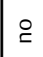 & 잉 & 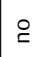 & ○ & 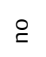 & 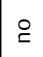 & $\stackrel{\circ}{\complement}$ & ○ & ○ & 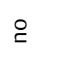 & $\circ$ & 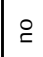 \\
\hline 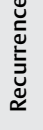 & $\stackrel{\circ}{\circ}$ & 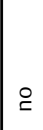 & $\stackrel{\circ}{\circ}$ & $\check{\check{\nu}}$ & 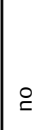 & 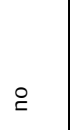 & $\stackrel{\circ}{\circ}$ & $\stackrel{\circ}{\circ}$ & $\stackrel{\circ}{\stackrel{2}{2}}$ & $\stackrel{\circ}{\stackrel{\circ}{2}}$ & $\stackrel{\circ}{\circ}$ & 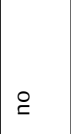 & 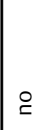 & 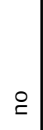 & 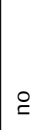 & 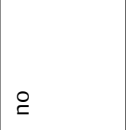 & $\stackrel{\circ}{\check{2}}$ & 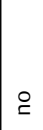 & 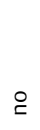 & 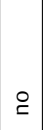 & 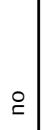 & 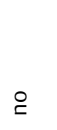 & 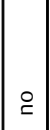 & $\stackrel{\circ}{\check{2}}$ \\
\hline 근 & $\bar{z}$ & 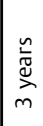 & 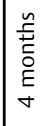 & 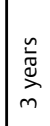 & $\bar{z}$ & 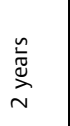 & $\begin{array}{l}\stackrel{\sim}{\bar{n}} \\
\stackrel{\Delta}{\sim} \\
\sim\end{array}$ & 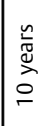 & $\bar{z}$ & $\bar{z}$ & $\bar{z}$ & $\bar{z}$ & $\bar{z}$ & $\bar{z}$ & 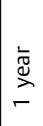 & 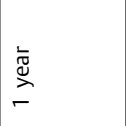 & $\bar{z}$ & 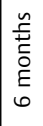 & ${ }_{\infty}^{c}$ & $\bar{z}$ & $\bar{z}$ & 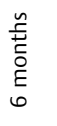 & $\bar{z}$ & 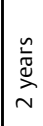 \\
\hline$\underline{\underline{\underline{I}}}$ & $\begin{array}{l}0 \\
\\
+ \\
8 \\
\dot{\sigma} \\
\dot{n}\end{array}$ & $\bar{z}$ & \begin{tabular}{|l}
0 \\
\\
+ \\
8 \\
$\dot{\sigma}$ \\
$\dot{\omega}$
\end{tabular} & $\bar{z}$ & \begin{tabular}{|l}
0 \\
\\
+ \\
8 \\
$\dot{0}$ \\
$\dot{n}$
\end{tabular} & 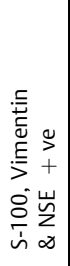 & \begin{tabular}{|l|} 
\\
$z$ \\
+ \\
8 \\
$\dot{\sigma}$ \\
$\dot{u}$
\end{tabular} & $\bar{z}$ & $\bar{z}$ & \begin{tabular}{|l}
$\tilde{E}$ \\
$\sum_{\infty}$ \\
$\infty$
\end{tabular} & $\bar{z}$ & 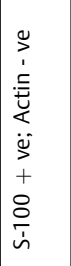 & $\bar{z}$ & 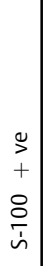 & $\bar{z}$ & 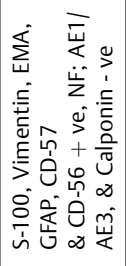 & $\bar{z}$ & 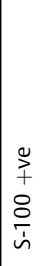 & $\bar{z}$ & $\bar{z}$ & $\begin{array}{l}0 \\
+ \\
+ \\
0 \\
\vdots \\
\dot{n}\end{array}$ & $\begin{array}{l}\stackrel{0}{2} \\
+ \\
8 \\
\vdots \\
\dot{n}\end{array}$ & $\mid \begin{array}{l}0 \\
+ \\
+ \\
o \\
\dot{v} \\
\dot{\omega}\end{array}$ & \begin{tabular}{|l} 
\\
\\
+ \\
$\dot{0}$ \\
$\dot{\square}$ \\
$\dot{n}$
\end{tabular} \\
\hline
\end{tabular}

\begin{tabular}{|c|c|c|c|c|c|c|c|c|c|c|c|c|c|c|c|c|c|c|c|c|}
\hline 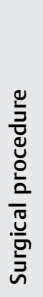 & z & 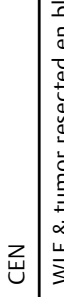 & 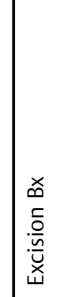 & 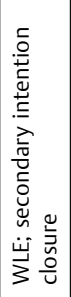 & 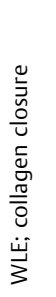 & 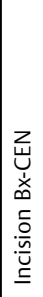 & 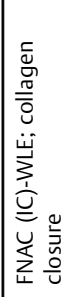 & 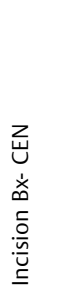 & 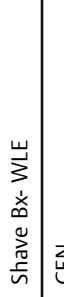 & $\vec{u} \mid \vec{J}$ & 恙 & 至 & 胥 & 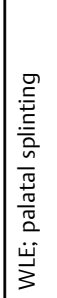 & 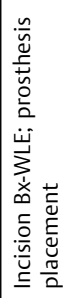 & 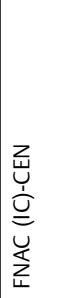 & 런 & $\vec{\xi}$ & 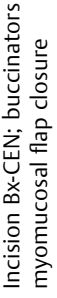 & 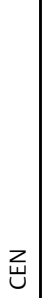 \\
\hline 站 & 产 & $\overline{\bar{\alpha}}$ & 至 & 害 & $\frac{\rho}{\underline{T}}$ & 至 & 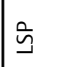 & 当 & \begin{tabular}{l|l}
$\frac{a}{\Sigma}$ & \\
$\frac{1}{2}$
\end{tabular} & 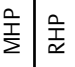 & $\frac{9}{\frac{9}{\alpha}}$ & 言 & $\hat{\underline{\aleph}}$ & $\mid \Sigma$ & & 变 & 产 & n & $\hat{\underline{\aleph}}$ & $\hat{\grave{\alpha}}$ \\
\hline
\end{tabular}

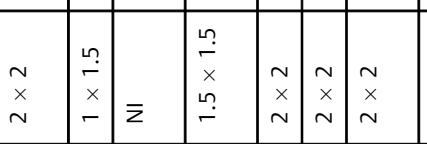

\begin{tabular}{|c|c|c|c|c|c|c|c|c|c|c|c|c|c|c|c|c|c|c|c|c|c|c|c|c|}
\hline 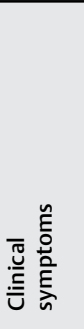 & 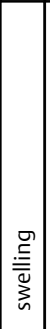 & 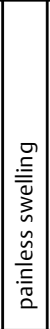 & $\mid \begin{array}{l}\text { 产 } \\
\underline{\underline{E}}\end{array}$ & 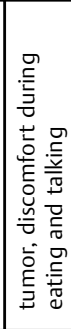 & 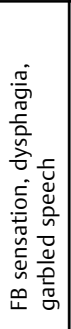 & 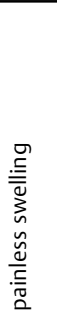 & 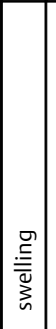 & 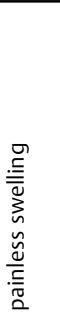 & 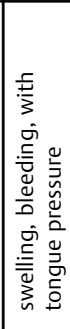 & 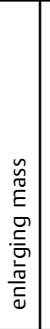 & 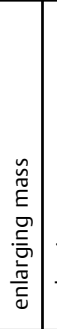 & 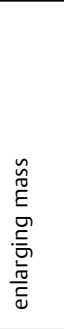 & 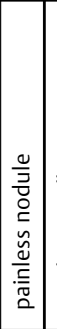 & 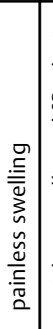 & 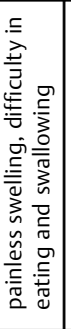 & 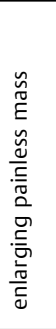 & 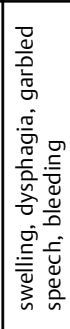 & 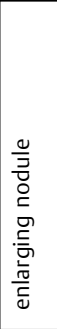 & 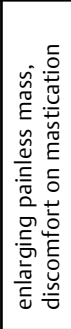 & 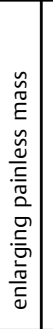 & 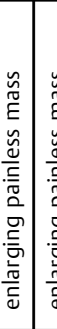 & & & 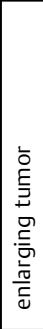 \\
\hline 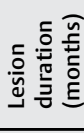 & $\bar{z}$ & $\stackrel{ \pm}{\sim}$ & $\sigma$ & $\simeq$ & $m$ & $m$ & $m$ & 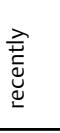 & 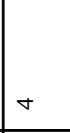 & $m$ & $\stackrel{\infty}{m}$ & $\stackrel{\infty}{\leftarrow}$ & 8 & 0 & 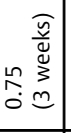 & 0 & in & $\stackrel{\sim}{\sim}$ & i & $\approx$ & \pm & $m$ & - & 8 \\
\hline $\begin{array}{l}\text { Zे } \\
\text { 产 }\end{array}$ & \begin{tabular}{|l|} 
\\
0 \\
0 \\
0 \\
0
\end{tabular} & 弚 & \begin{tabular}{|l}
$\frac{\pi}{0}$ \\
元 \\
\end{tabular} & 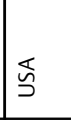 & 经 & 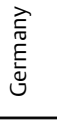 & \begin{tabular}{|l|} 
\\
$\overline{\bar{n}}$ \\
\end{tabular} & $\begin{array}{l}\stackrel{\underline{\underline{v}}}{\underline{\underline{E}}} \\
\end{array}$ & \begin{tabular}{|l} 
吾 \\
$\underline{\underline{\underline{E}}}$
\end{tabular} & 岕 & $\begin{array}{l}\frac{\sqrt[\sigma]{0}}{\underline{\underline{\underline{v}}}} \\
\text {. }\end{array}$ & $\begin{array}{l}\text { 离 } \\
\text { 咅 }\end{array}$ & \begin{tabular}{|l|}
$\overline{\bar{N}}$ \\
$\overline{0}$ \\
0 \\
\end{tabular} & 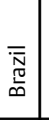 & 兰 & 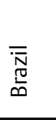 & 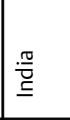 & 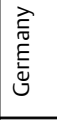 & \begin{tabular}{|l}
$\stackrel{\underline{\underline{\sigma}}}{\underline{\underline{\underline{E}}}}$ \\
\end{tabular} & 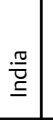 & 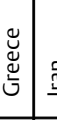 & 름 & \begin{tabular}{|l}
$\frac{\sqrt{\underline{\tau}}}{\underline{\underline{\underline{5}}}}$ \\
\end{tabular} & 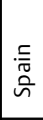 \\
\hline 岛 & $\frac{u}{\sigma}$ & $\frac{\mathrm{L}}{\mathrm{N}}$ & $\sum_{\vec{f}}$ & $\underset{\sim}{\stackrel{u}{\sim}}$ & 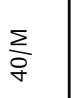 & $\stackrel{\stackrel{u}{\Xi}}{\underline{\underline{u}}}$ & 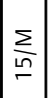 & $\frac{u}{\vec{g}}$ & $\underset{\substack{\Delta \\
\sim}}{\sim}$ & $\underset{m}{m}$ & $\frac{u}{\vec{J}}$ & 岁 & 峪 & $\frac{u}{\hat{m}}$ & $\sum_{\sigma}$ & $\frac{\omega}{m}$ & $\stackrel{\sum}{0}$ & 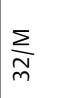 & $\frac{\mathrm{L}}{2}$ & $\stackrel{E}{\infty}$ & $\stackrel{\vec{\sim}}{\mathbf{N}}$ & $\frac{\underline{U}}{\underline{\underline{U}}}$ & $\frac{\sum}{\tilde{\gamma}}$ & 岕 \\
\hline 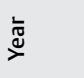 & 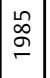 & $\mid \begin{array}{l}\infty \\
\stackrel{\infty}{\sigma} \\
\stackrel{2}{2}\end{array}$ & $\mid \begin{array}{l}\mathfrak{\infty} \\
\stackrel{\infty}{\Im}\end{array}$ & 声 & ठั & 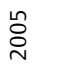 & $\mid \begin{array}{l}\text { :े } \\
\text { iे }\end{array}$ & : & 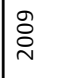 & $\begin{array}{l}0 \\
\vdots \\
\vdots\end{array}$ & $\begin{array}{l}0 \\
\vdots \\
\vdots \\
\sim\end{array}$ & $\stackrel{\circ}{\stackrel{\sim}{\sim}}$ & $\left(\begin{array}{l}0 \\
\vdots \\
\vdots\end{array} \mid\right.$ & $\begin{array}{l}0 \\
\vdots \\
\vdots\end{array}$ & $\bar{i}$ & $\bar{i}$ & $\frac{\sim}{i}$ & $\frac{\tilde{N}}{\dot{N}}$ & 烃 & 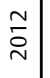 & 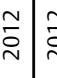 & 文 & 爫 & $\stackrel{m}{i}$ \\
\hline 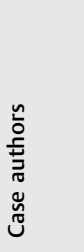 & 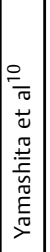 & 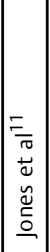 & 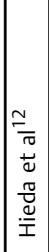 & 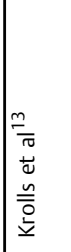 & 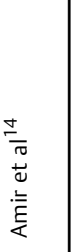 & 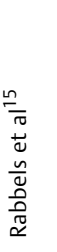 & 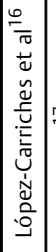 & 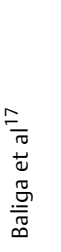 & 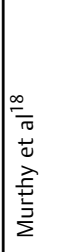 & $\begin{array}{l}\frac{9}{\overline{0}} \\
+ \\
\pm \\
\frac{5}{0} \\
\bar{\sigma}\end{array}$ & 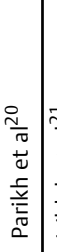 & 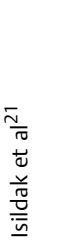 & 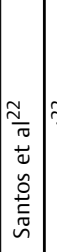 & 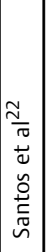 & 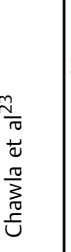 & 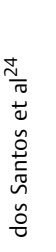 & 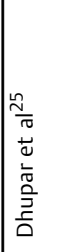 & 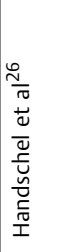 & 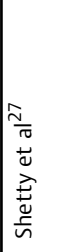 & 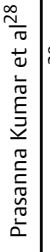 & 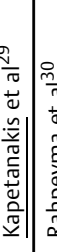 & 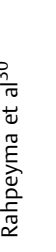 & 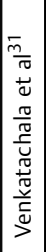 & 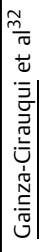 \\
\hline
\end{tabular}




\begin{tabular}{|c|c|c|c|c|c|c|c|c|c|c|c|c|c|c|c|c|c|c|c|c|c|c|c|}
\hline 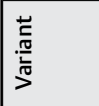 & Z & z & Z & 2 & 2 & Z & Z & Z & 2 & Z & z & z & 3 & 2 & U & 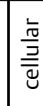 & 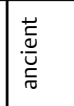 & Z & 2 & 2 & 2 & 2 & 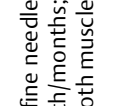 \\
\hline$\Sigma$ & 용 & $\div$ & 요 & $\circ$ & 웅 & 웅 & 욤 & 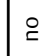 & 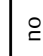 & 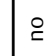 & 웅 & 욤 & ㅇ & 잉 & 요 & ㅇ & 응 & 응 & : & 잉 & 잉 & ○ & \\
\hline $\mid \begin{array}{l}\vec{Z} \\
\check{a}\end{array}$ & 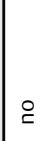 & ㅇ & 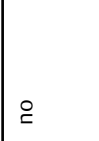 & 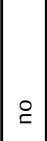 & 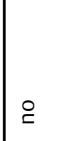 & 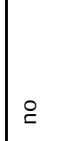 & 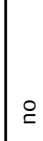 & 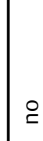 & 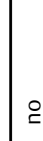 & 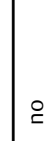 & 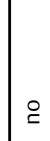 & ○ & 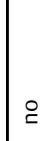 & 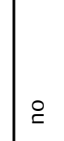 & 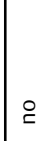 & ঃ & 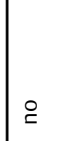 & 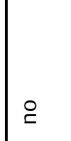 & 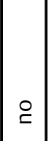 & 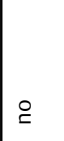 & 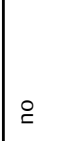 & 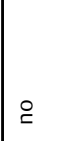 & 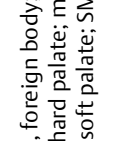 \\
\hline$=$ & $\bar{z}$ & 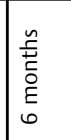 & 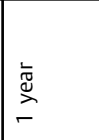 & $\bar{z}$ & 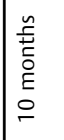 & 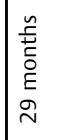 & 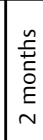 & $\begin{array}{l}\text { 志 } \\
\text { 言 } \\
\underline{E}\end{array}$ & $\bar{z}$ & 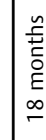 & $\bar{z}$ & 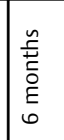 & 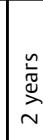 & $\mid \begin{array}{l}\frac{n}{0} \\
\frac{\hat{N}}{\Sigma}\end{array}$ & 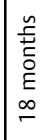 & $\begin{array}{l}\text { 矛 } \\
\stackrel{-}{二}\end{array}$ & 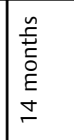 & 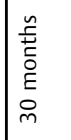 & 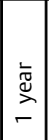 & $\bar{z}$ & 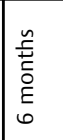 & 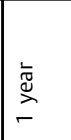 & $\underbrace{\bar{E}}$ \\
\hline 至 & $\bar{z}$ & $\begin{array}{l}8 \\
\\
+ \\
8 \\
0 \\
\dot{1} \\
\end{array}$ & $\bar{z}$ & $\bar{z}$ & 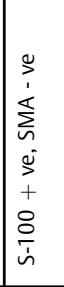 & 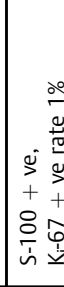 & $\bar{z}$ & $\bar{z}$ & $\bar{z}$ & 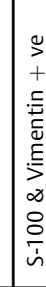 & $\bar{z}$ & \begin{tabular}{|l}
0 \\
$\vdots$ \\
+ \\
0 \\
$\vdots$ \\
$\dot{n}$ \\
\end{tabular} & $\begin{array}{l}8 \\
+ \\
+ \\
8 \\
\dot{n}\end{array}$ & 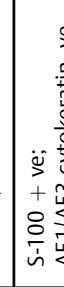 & $\bar{z}$ & $\begin{array}{l}8 \\
+ \\
+ \\
8 \\
\dot{v} \\
\end{array}$ & 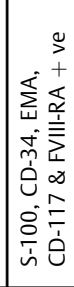 & $\bar{z}$ & 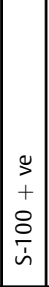 & 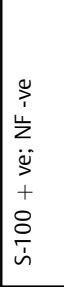 & \begin{tabular}{|l}
0 \\
2 \\
+ \\
0 \\
$\vdots$ \\
$\dot{n}$
\end{tabular} & 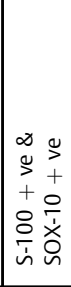 & 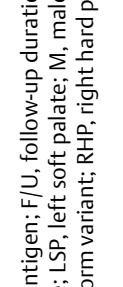 \\
\hline 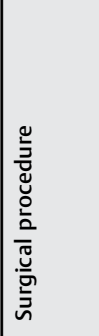 & 出 & 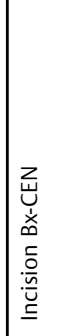 & 出 & 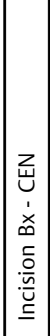 & $\mid$ & 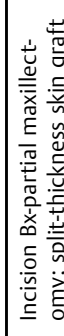 & 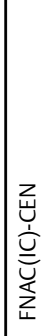 & $\vec{\xi}$ & 总 & 出 & 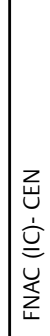 & 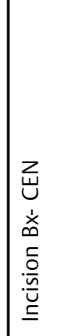 & 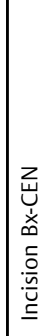 & 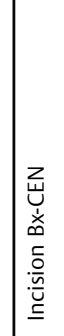 & 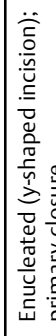 & 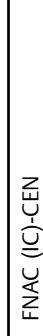 & 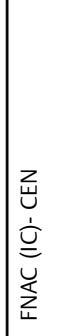 & 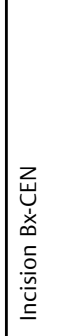 & 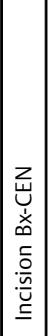 & 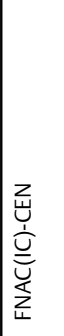 & 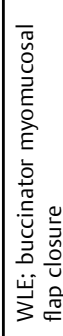 & z & 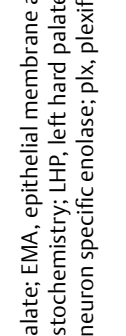 \\
\hline$\stackrel{ \pm}{n}$ & 至 & 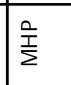 & 至 & 产 & 至 & 主 & 至 & 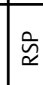 & $\overline{\grave{\alpha}}$ & 产 & $\frac{a}{\alpha}$ & 乡 & 产 & जิ & 产 & 产 & जิ & $\bar{\alpha}$ & $\frac{\stackrel{p}{a}}{\alpha}$ & 善 & $\frac{\hat{n}}{\Sigma}$ & जิ & \\
\hline 듬음 & $\begin{array}{l}\sim \\
\stackrel{x}{*} \\
\sim\end{array}$ & $\begin{array}{l}\sim \\
\times \\
\sim\end{array}$ & $\begin{array}{l}m \\
x \\
\sigma \\
\end{array}$ & \begin{tabular}{|l}
$\stackrel{n}{\leftarrow}$ \\
$\times$ \\
$\sim$ \\
$\sim$
\end{tabular} & m & m & $\begin{array}{l}m \\
\stackrel{m}{x} \\
-\end{array}$ & - & $\begin{array}{r}+ \\
\stackrel{+}{+}\end{array}$ & $\begin{array}{l}\text { in } \\
\stackrel{x}{m}\end{array}$ & $\begin{array}{l}m \\
x \\
\sim\end{array}$ & $\stackrel{\text { In }}{=}$ & N & $\mid \begin{array}{l}\tilde{N} \\
x \\
\underline{-} \\
\stackrel{-}{-}\end{array}$ & $\sim$ & $\begin{array}{r}-\bar{x} \\
-\end{array}$ & $\begin{array}{l}\tilde{N} \\
\times \\
m\end{array}$ & $\begin{array}{l}\alpha \\
x \\
\sim\end{array}$ & \begin{tabular}{|c|}
$m$ \\
$x$ \\
$n$ \\
$m$ \\
$m$
\end{tabular} & $\begin{array}{l}m \\
x \\
\sim\end{array}$ & $\begin{array}{l}\frac{g}{2} \\
\times \\
\sim\end{array}$ & $\mid \begin{array}{l}\tilde{N} \\
x \\
n \\
\end{array}$ & \\
\hline 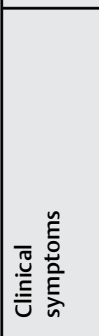 & $\cong$ & 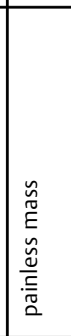 & 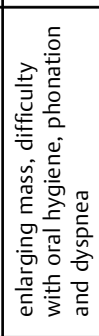 & 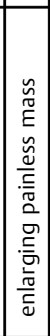 & 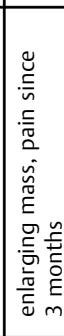 & $\cong$ & $\cong$ & 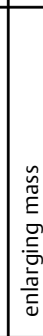 & 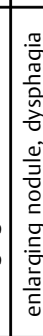 & 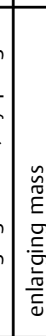 & 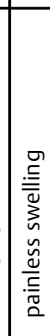 & 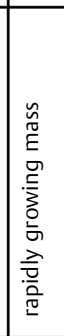 & 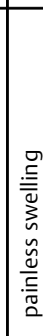 & o & $\cong$ & 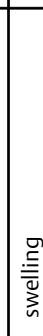 & 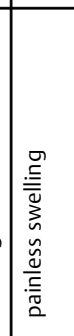 & 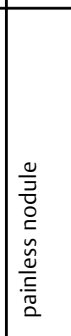 & 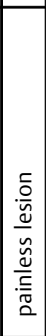 & 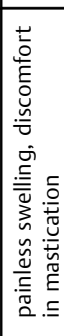 & 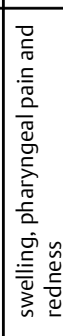 & 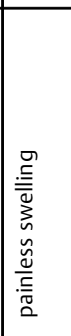 & 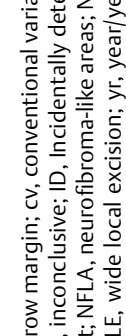 \\
\hline 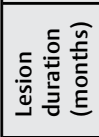 & $\cong$ & N & $\underset{d}{\sim}$ & $\simeq$ & $\stackrel{\infty}{q}$ & $\cong$ & $\cong$ & $\simeq$ & $\simeq$ & $\stackrel{i}{2}$ & $\simeq$ & 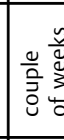 & 0 & $\cong$ & 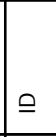 & $\infty$ & $\stackrel{\circ}{\wedge}$ & 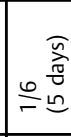 & i & $\stackrel{\infty}{\sim}$ & $\simeq$ & $\sim$ & \\
\hline 居 & \begin{tabular}{|l}
$\frac{\sqrt{0}}{\underline{\underline{T}}}$ \\
$\underline{\underline{\underline{5}}}$
\end{tabular} & 胥 & 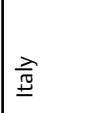 & 竞 & 吾 & \begin{tabular}{|l}
$\frac{\sqrt{0}}{2}$ \\
㒸
\end{tabular} & \begin{tabular}{|l}
$\frac{\pi}{\bar{t}}$ \\
$\underline{\underline{\underline{T}}}$
\end{tabular} & 孚 & $\underline{\underline{\underline{\underline{\sigma}}}}$ & 总 & 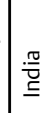 & $\frac{2}{\underline{\underline{e n}}}$ & 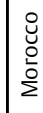 & 孚 & ১ & 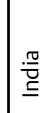 & $\begin{array}{l}\text { 㖣 } \\
\text { ñ }\end{array}$ & 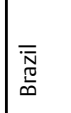 & 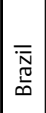 & 尝 & 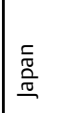 & 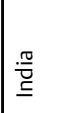 & \\
\hline 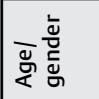 & $\frac{u}{\widetilde{\gamma}}$ & $\underset{\sim}{\grave{N}}$ & $\frac{u}{g}$ & $\underset{m}{\frac{u}{F}}$ & $\underset{\substack{\infty \\
\sim}}{\sim}$ & $\underset{\infty}{\infty}$ & 訔 & $\stackrel{\mathrm{u}}{\frac{\mathrm{U}}{5}}$ & $\stackrel{\sum}{\infty}$ & 惢 & $\frac{\mathrm{u}}{6}$ & $\frac{u}{m}$ & $\sum_{m}$ & $\begin{array}{l}\text { 岕 } \\
\text { 足 }\end{array}$ & & 訔 & $\frac{\mathrm{u}}{\mathrm{s}}$ & $\underset{\substack{0 \\
N}}{\sum}$ & \begin{tabular}{|l}
$E$ \\
$\stackrel{E}{\infty}$ \\
-
\end{tabular} & $\frac{u}{m}$ & $\frac{\omega}{\underline{I}}$ & $\frac{\omega}{6}$ & \\
\hline ¿ & $\stackrel{m}{\stackrel{n}{n}}$ & $\frac{\pi}{\stackrel{N}{~}}$ & $\frac{\vec{\sigma}}{\stackrel{N}{n}}$ & 䓂 & 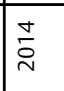 & $\stackrel{n}{\stackrel{n}{N}}$ & $\stackrel{n}{\stackrel{n}{2}}$ & $\stackrel{n}{\stackrel{n}{n}}$ & $\stackrel{n}{\stackrel{n}{\sim}}$ & $\stackrel{n}{n}$ & $\stackrel{n}{\stackrel{n}{\sim}}$ & $\stackrel{n}{\stackrel{n}{\sim}}$ & $\frac{0}{\stackrel{2}{2}}$ & $\frac{0}{2}$ & $\vec{\Xi}$ & $\hat{\stackrel{n}{n}}$ & $\overline{\grave{N}}$ & $\stackrel{\sim}{\stackrel{N}{2}}$ & 立 & 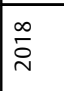 & 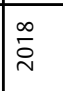 & i & \\
\hline 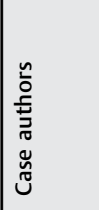 & 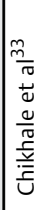 & 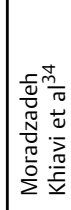 & $\begin{array}{l}\frac{m}{\sqrt{0}} \\
\overrightarrow{0} \\
\frac{1}{0} \\
\frac{0}{2}\end{array}$ & 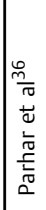 & 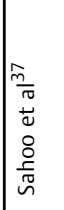 & 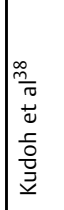 & 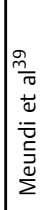 & 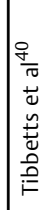 & 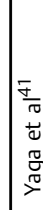 & 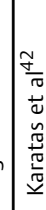 & 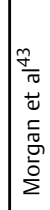 & 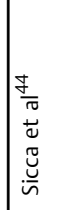 & 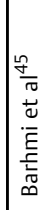 & $\frac{10}{\frac{\pi}{0}}$ & 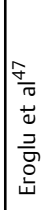 & 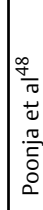 & 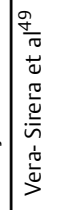 & 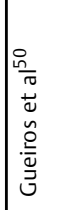 & 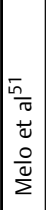 & 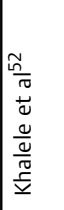 & 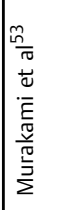 & 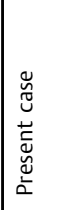 & 竘 \\
\hline
\end{tabular}


Table 2 Updated clinical profile of reported cases of palatal schwannomas

\begin{tabular}{|c|c|c|}
\hline \multicolumn{3}{|c|}{ Clinical features of 46 patients with palatal schwannomas } \\
\hline Feature & Data $^{a}$ & Number of cases amenable to analysis \\
\hline Female & $29(64 \%)$ & 45 \\
\hline Mean age at time of initial evaluation, years old & 30.04 (range: $3-84$ ) & 46 \\
\hline Mean duration of lesion, months & 25.63 (range: 5 days- 20 years) & 38 \\
\hline Cases reported from India & $15(32.6 \%)$ & 46 \\
\hline Hard palate involvement & $31(67.4 \%)$ & 46 \\
\hline Soft palate involvement & $15(32.6 \%)$ & 46 \\
\hline Mean size of lesion, centimeters & 2.4 (range: $1-5$ ) & 44 \\
\hline Symptomatic cases & $41(89 \%)$ & 46 \\
\hline Asymptomatic cases/ cases detected incidentally & $5(11 \%)$ & 46 \\
\hline \multicolumn{3}{|l|}{ Symptoms at initial presentation } \\
\hline Painful lesion/ pharynx pain & $2(4.3 \%)$ & 46 \\
\hline Dysphagia & $5(10.9 \%)$ & 46 \\
\hline Dysphonia & $3(6.5 \%)$ & 46 \\
\hline Dyspnea & $1(2.2 \%)$ & 46 \\
\hline Difficulty in mastication & $4(8.7 \%)$ & 46 \\
\hline Bleeding from tumor & $2(4.3 \%)$ & 46 \\
\hline Foreign body sensation & $1(2.2 \%)$ & 46 \\
\hline Pharyngeal erythema & $1(2.2 \%)$ & 46 \\
\hline \multicolumn{3}{|l|}{ Signs at initial presentation } \\
\hline Tenderness over lesion & $2(4.3 \%)$ & 46 \\
\hline Soft on palpation & $4(8.7 \%)$ & 46 \\
\hline \multicolumn{3}{|l|}{ Histological variant } \\
\hline Conventional & $40(87 \%)$ & 46 \\
\hline Plexiform & $3(6.5 \%)$ & 46 \\
\hline Ancient & $2(4.3 \%)$ & 46 \\
\hline Cellular & $1(2.2 \%)$ & 46 \\
\hline \multicolumn{2}{|l|}{ IHC positive staining } & \multirow[t]{9}{*}{$27^{b}$} \\
\hline S-100 & $27(100 \%)$ & \\
\hline Vimentin & $3(11.1 \%)$ & \\
\hline EMA & $2(3.7 \%)$ & \\
\hline SOX-10 & $1(3.7 \%)$ & \\
\hline NSE & $1(3.7 \%)$ & \\
\hline GFAP & $1(3.7 \%)$ & \\
\hline CD-56 \& CD-57 & $1(3.7 \%)$ & \\
\hline CD-34 \& CD-117 & $1(3.7 \%)$ & \\
\hline \multicolumn{2}{|l|}{ Surgical treatment } & \multirow[t]{5}{*}{46} \\
\hline $\begin{array}{l}\text { Enucleation/complete excision with } \\
\text { narrow margin/excision biopsy }\end{array}$ & $33(71.7 \%)$ & \\
\hline Wide margin excision & $11(24 \%)$ & \\
\hline Partial maxillectomy & $1(2.2 \%)$ & \\
\hline En bloc resection & $1(2.2 \%)$ & \\
\hline \multicolumn{2}{|l|}{ Prognosis } & \multirow[t]{3}{*}{46} \\
\hline Recurrence & $1(2.2 \%)$ & \\
\hline Malignant transformation & $0(0 \%)$ & \\
\hline
\end{tabular}

Abbreviations: EMA, epithelial membrane antigen; GFAP, glial fibrillary acidic protein; SOX-10, SRY-related HMG-box 10.

${ }^{\mathrm{a}}$ Number (\%) unless otherwise specified.

${ }^{\mathrm{b}}$ Number of cases in which some form of immunohistochemistry staining was performed. 


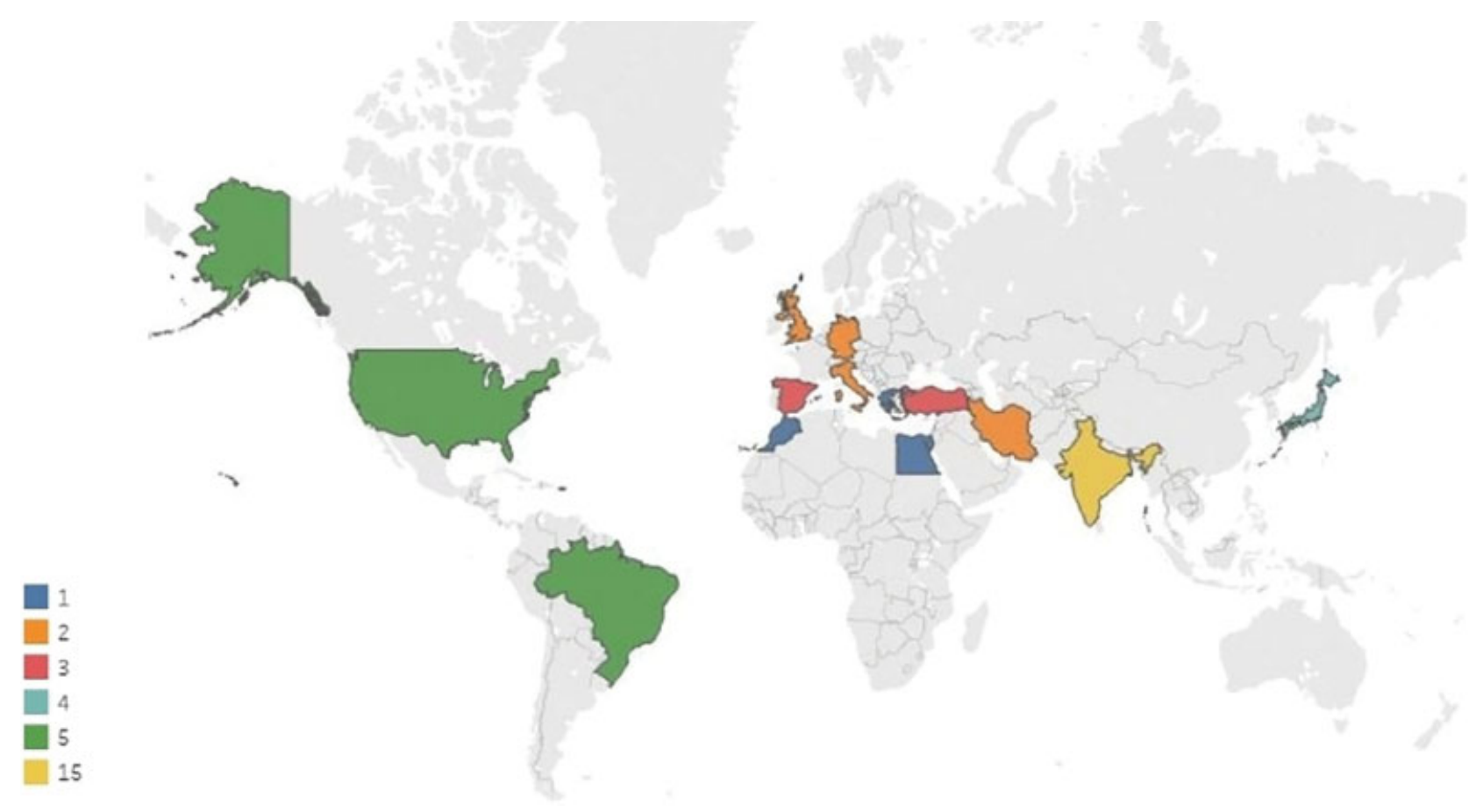

Fig. 7 Worldwide distribution of reported cases of palatal schwannomas.

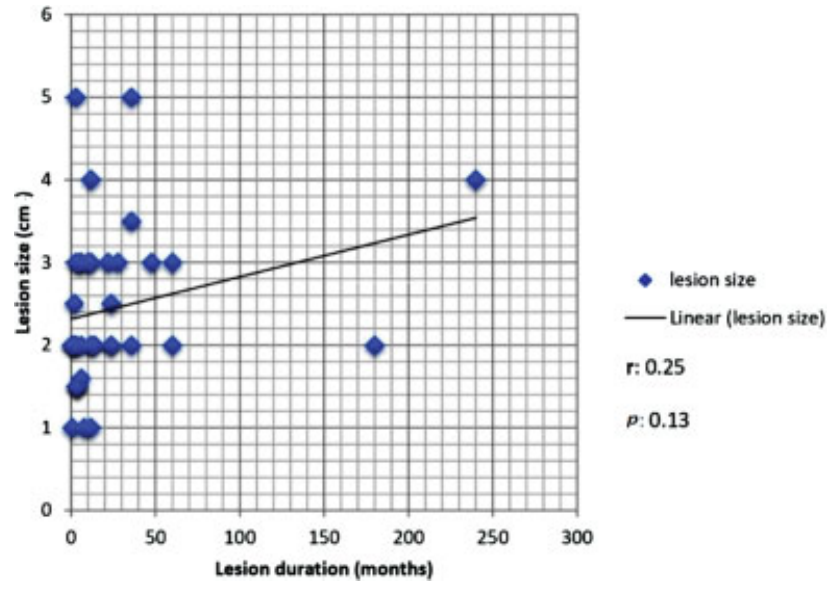

Fig. 8 Relationship between lesion size and duration of lesion.

pharyngeal pain was present. $^{53}$ Other symptoms were reported in the following frequency: dysphagia $(n=5)$ $14,23,25,31,41$ dysphonia/garbled speech $(n=3)^{14,25,35}$, dyspnea $(n=1)^{35}$, difficult mastication $(n=4)^{13,23,27,52}$, occasional bleeding $(\mathrm{n}=2)^{18,25}$, foreign body sensation $(n=1)$ ${ }^{14}$, difficulty in oral hygiene $(n=1)^{35}$ and pharyngeal redness $(n=1)^{53}$.

Clinically, most of the lesions were reported as nontender and/or firm/hard. Tenderness was elicited only twice ${ }^{21,37}$, while soft swelling on palpation was reported in four cases $18,38,43,44$. Jones et al reported a cystic component in the lesion. ${ }^{11}$ Most of the lesions had a healthy overlying mucosa without any obvious ulceration. Ulceration of the overlying mucosa was noted in seven other cases apart from present case. $^{13,23,30,31,34,37,44}$ The low frequency of ulceration reflects a good encapsulation of this tumor.
Of the histological variant, the conventional subtype dominated and was reported in 40 cases (87\%). It was followed by plexiform-3, ${ }^{13,24,29}$ ancient- $2,{ }^{32,49}$ and cellular variant- $1 .{ }^{48}$ Almost all of the cases with conventional phenotype exhibited Verocay bodies and a predominance of Antoni A areas over Antoni B areas. The additional presence of acute and chronic inflammatory infiltrate, ${ }^{23}$ areas of hyalinization, and thin blood vessels with/without thrombus/fibrin, ${ }^{11,28,30,31,46}$ and areas containing epithelioid cells have also been reported from cases with conventional phenotypes. ${ }^{46}$ In one report, a note was made about the predominance of neurofibroma-like areas. ${ }^{21}$

Various IHC staining were performed in 27 patients. S-100 staining was employed in all of the 27 amenable cases, and showed strong immunoreactivity in all of the employed cases. Other authors revealed varying degrees of positive immunoreactivity with different stains: vimentin, ${ }^{15,19,42}$ epithelial membrane antigen (EMA), ${ }^{24,49}$ soX-10 (present case), neuron specific enolase (NSE), ${ }^{15}$ glial fibrillary acidic protein (GFAP), ${ }^{24}$ CD-56 and CD-57, ${ }^{24}$ and CD-34 and CD117. ${ }^{49}$ Immunonegative results were noted with the following stains: actin, ${ }^{21}$ neurofilament protein (NFP), ${ }^{24,52}$ smooth muscle antigen, ${ }^{37}$ cytokeratin, ${ }^{46} \mathrm{AE} 1 / \mathrm{AE} 3,{ }^{24}$ and Calponin. ${ }^{24}$

Various imaging modalities capable of revealing abnormal palatal morphologies were performed on 36 patients, including simple X-ray scan in 1, panoramic radiography (orthopantomogram [OPG]) in 6, maxillary occlusal radiography in 7, CT scan (with or without contrast) in 20 , magnetic resonance imaging (MRI) in 7, and positron emission tomography (PET) scan in 1 patient. The principal CT scan finding was an isodense-hypodense soft tissue lesion without any bony erosion/resorption. Partial/complete bony erosion was noted in 6 instances. $^{15,16,33,37,38,51}$ The lesion was seen 
eroding into the right maxillary sinus through the floor of the sinus in one case; ${ }^{15}$ while in another patient, the mass was seen invading the nasal cavity through the palatal bone. ${ }^{38}$ Orthopantomogramand maxillary occlusal radiography showed mostly a radiolucent lesion, without any bony alteration or periapical changes. In MRI exams, the mass appeared hypo-to-isointense on T1-weighted images and hyperintense on $\mathrm{T} 2$-weighted images, in almost all of the cases. Small foci of central calcification were evident on MRI in one occasion. ${ }^{40}$

With the exception of two cases, all of the lesions were treated with simple surgical removal, either with enucleation, with wide local excision with a good margin, or with a complete resection without incorporating a wide margin. The remaining two cases were treated by partial maxillectomy $^{38}$ and by en bloc resection. ${ }^{12}$ The defect was either closed primarily or was allowed to heal by secondary intention. Otherwise, large defects were closed with some form of prosthesis, splints, grafts or flaps. Reconstruction using buccinator myomucosal pedicle flap, ${ }^{30,53}$ split-thickness skin graft, ${ }^{38}$ palatal splint, ${ }^{25}$ and collagen sheet have been reported by various authors. ${ }^{15,17}$

Preoperative fine-needle aspiration cytology (FNAC) was performed in 10 cases, while incisional biopsy was performed in 13 cases. Fine-needle aspiration cytology was inconclusive in almost all of the cases.

Postoperative follow-up was performed in 29 cases, and the follow-up duration ranged from 11 days to a decade. Recurrence was noted only once, ${ }^{13}$ while none of the included cases reported malignant transformation.

\section{Discussion}

Schwannoma is synonymous with neurinoma, neurilemmoma, and perineural fibroblastoma. ${ }^{22}$ It arises from cranial, peripheral, or autonomic nerves that contain Schwann cells. It never arises from cranial nerves I and II, since they lack Schwann cells. ${ }^{16}$ Sensory nerve is more common, with rare involvement of motor nerve. ${ }^{54}$

About 25 to $45 \%$ of all schwannomas are found in the head and neck region, and only between 1 to $12 \%$ of them have an intraoral origin. ${ }^{7-9}$ However, the palatal location is rare. In a review of 52 cases of schwannomas of the head and neck region, only 1 case of schwannoma over the hard palate was reported. ${ }^{55}$ A review of the literature on oral, as well as on head and neck schwannomas, showed varying results about the gender predilection of the tumor. Williams et al showed a male predominance of the tumor; for Lucas et al, there was a greater predilection for females, ${ }^{56,57}$ while other authors reported no gender predilection. ${ }^{58,59}$ Although reported in all age groups, schwannomas are more common in the $2^{\text {nd }}$ and $3^{\text {rd }}$ decades of life. ${ }^{21}$

Intraoral and palatal schwannomas are mostly solitary lesions. ${ }^{34}$ Multiple nerve schwannomas require evaluation for Von-Reklinghausen disease, while bilateral vestibular schwannomas raise suspicion for neurofibromatosis-II. ${ }^{30}$ The majority of palatal schwannomas have been reported on the lateral aspect of the palate.
Based on its location, schwannomas have been classified either as central (bone) or peripheral (soft tissue) type. The tumor may arise centrally in the bone, may arise within the nutrient canal, or a soft tissue tumor may secondarily erode into the bony tissue. ${ }^{60}$ There are two clinical forms of oral schwannomas: the encapsulated form, surrounded by dense fibrous connective tissue, and the pediculate/nonencapsulated form, in which the tumor is located just below the mucous membrane. ${ }^{61}$

Although the etiology of schwannomas is unknown, trauma is considered to be an unclear etiological cause. ${ }^{2}$ There are various theories about its onset: 1) ectodermal tumor derived from Schwann cells; and 2) mesodermal tumor arising from the perineurium. ${ }^{38}$

Most of the cases are asymptomatic, while most of the lesions are slow-growing. A sudden increase in size may be due to internal hemorrhage. ${ }^{16}$ The clinical presentation depends upon the site of the tumor, the size of the tumor, and upon the anatomy of the affected nerve. ${ }^{32}$

There are four major histological types of schwannoma: conventional, plexiform, cellular, and ancient variant. According to Erlandson, schwannomas are classified into seven subtypes: conventional, cellular, plexiform, cranial nerve, melanotic, ancient, and granular cell schwannomas. ${ }^{62}$ However, they have mainly two distinct histological patterns: Antoni types A and B. Antoni patterns were first described by Prof. Nils Ragnar Eugene Antoni. Antoni A areas consist of a hypercellular proliferation of fusiform cells, often arranged in a palisading pattern around a central acellular eosinophilic area known as Verocay bodies, while Antoni B areas are hypocellular and less organized.

The conventional variant consists mostly of Antoni A areas and Verocay bodies, with the occasional presence of Antoni B areas. The additional presence of acute/chronic inflammatory infiltrate, areas of hyalinization, and thin vessels containing thrombin are noted in the conventional variant. ${ }^{22}$ The cellular variant is characterized by a marked increase in cellularity, with a compact arrangement of spindle cells in fascicles, variable nuclear hyperchromasia and pleomorphism, lack of Verocay bodies, and a predominance of Antoni A areas. $^{48,64}$ The cellular variant, due to the increased mitotic activity and to the high potential for body destruction, is often confused with sarcoma. ${ }^{65}$

The ancient variant is characterized by degenerative changes, such as calcification, mild pleomorphism and bizarre nuclei, microcyst formation, dilated vessels, and hemorrhagic phenomena. Some authors believe that the absence of symptoms and the long history of the lesion are the probable cause of transformation into an ancient variant. $^{32}$ The plexiform type consists of both Antoni A and B regions with prominent Verocay bodies, like the conventional variant; however, the Schwann cells show a nodular arrangement with capsular delineation. ${ }^{24}$

Immunohistochemistry is important to distinguish schwannoma from other close differentials, and can aid in its diagnosis; however, it is not mandatory to confirm the diagnosis. S-100 is undoubtedly the first 
immunostaining that comes into mind when dealing with suspected peripheral nerve tumors. Both schwannomas and neurofibromas show moderate to strong reactivity to S-100. However, S-100 has low specificity for diagnosing peripheral nerve cell tumors. One study has found Sox-10 to be more sensitive and specific than S-100 for peripheral nerve tumors. ${ }^{66}$ Diffuse staining with $\mathrm{CD}-34$ is seen in neurofibromas, while schwannomas only occasionally show some focal staining in noncellular (Antoni-B) areas. Calretinin staining is found to be highly specific for schwannoma and useful in differentiating it from neurofibroma. ${ }^{67,68}$ Intensive staining with CD-57 is noted in traumatic neuromas. ${ }^{69}$ Schwannomas also stain positive with Leu-7antigen, GFAP, and vimentin. ${ }^{18}$ The presence of axons in palisaded encapsulated neuroma (PEN) and, therefore, positive staining with NFP, distinguishes it from schwannomas. ${ }^{70}$ Staining with AE1/AE3 and with calponin can help rule out salivary gland tumors. ${ }^{24}$

The major differentials are benign salivary gland tumors, benign peripheral nerve tumors (neurofibroma, traumatic neuroma, and PEN), other benign mesenchymal tumors (lipoma and hemangiomas), and odontogenic tumors. Salivary gland tumors are the most common differential in our review, and were considered in 25 cases (54.3\%).

Imaging modalities such as CT and MRI are useful during the initial workup to know the extent of the tumor, to delineate any bony erosion, to identify the nerve of origin, and to narrow the differentials. ${ }^{14,16,19,21}$ Yamazaki et al reported a case of a rapid growing lesion which was found in MRI to be originating from the mental nerve; therefore, the imaging exam assisted in the preoperative diagnosis of a peripheral nerve tumor that was otherwise considered a malignant lesion. ${ }^{71}$ Schwannomas mostly appear iso- to hypointense on T1-weighted MRI images and hyperintense on T2-weighed MRI images. Computed tomography scans generally show a well-circumscribed, soft tissue lesion without any bony erosion. However, schwannomas can occasionally cause pressure erosion of the bone. ${ }^{42,72}$ The proportion of Antoni-A and B areas has been reported to have a significant influence on the imaging findings. Gomez-Brouchet et al reported that vestibular schwannomas with a homogeneous appearance on MRI were predominantly made of Antoni-A tissues, while those with heterogeneous/cystic features were predominantly composed of Antoni-B/mixed tissues. $^{73}$

The therapy of choice consists of complete surgical removal. Schwannomas do not recur if they are completely removed. Only one case of benign palatal schwannoma has been found to recur after excision. ${ }^{13}$ Malignant transformation of head and neck schwannomas are exceedingly unusual, although it has been reported. ${ }^{74,75}$

\section{Final Comments}

There is barely any paper in the literature focusing extensively on palatal schwannomas, and tackling this benign tumor as an individual entity. Despite divergent inferences from different articles, the following conclusions can be drawn:

- Predominance in females, and involvement of the hard palate is twice the involvement of the soft palate.

- Although reported in all age groups, schwannomas commonly present during the $2^{\text {nd }}$ or $3^{\text {rd }}$ decades of life.

- Slow-growing tumor with a mean lesion duration of 25.63 months.

- Mostly present on the lateral aspect of the palate, with occasional reports of midpalatal or panpalatal lesions.

- Almost always a solitary tumor, presenting as a painless, nontender, and firm swelling.

- The conventional variant is the most common histological phenotype.

- Imaging can add to suspicion and can delineate a differential diagnosis, but the diagnosis is confirmed by pathological examination.

- Benign tumor of the salivary gland is the most common clinical differential.

- Fine-needle aspiration cytology is mostly inconclusive. Immunohistochemistry can assist in confirming a diagnosis, but is more helpful to rule out close differentials.

- Complete surgical removal is the treatment of choice.

- Recurrence and malignant transformation are extremely rare.

Conflicts of Interests

The authors have no conflicts of interests to declare.

\section{References}

1 Shah AA, Latoo S, Ahmad I, Malik AH, Singh AP, Hassan S. Schwannoma causing resorption of zygomatic arch. J Oral Maxillofac Pathol 2011;15(01):80-84

2 Pfeifle R, Baur DA, Paulino A, Helman J. Schwannoma of the tongue: report of 2 cases. J Oral Maxillofac Surg 2001;59(07): 802-804

3 Colreavy MP, Lacy PD, Hughes J, et al. Head and neck schwannomas-a 10 year review. J Laryngol Otol 2000;114(02):119-124

4 Verocay J.Zur Kenntnis der Neurofibrome. Beitr Pathol Anat 1910; 156:1-68

5 Masson P. Experimental and Spontaneous Schwannomas (Peripheral Gliomas): I. Experimental Schwannomas. Am J Pathol 1932;8(04):367-388.1, 1

6 Tarlov IM. Origin of perineural fibroblastoma. Am J Pathol 1940; 16(01):33-40, 7

7 Buric N, Jovanovic G, Pesic Z, et al. Mandible schwannoma (neurilemmoma) presenting as periapical lesion. Dentomaxillofac Radiol 2009;38(03):178-181

8 Subhashraj K, Balanand S, Pajaniammalle S. Ancient schwannoma arising from mental nerve. A case report and review. Med Oral Patol Oral Cir Bucal 2009;14(01):E12-E14

9 Jahanshahi G, Haghighat A, Azmoodeh F. Intraosseous neurilemmoma of the mandible: report of a rare ancient type. Dent Res J (Isfahan) 2011;8(03):150-153

10 Yamashita N, Kameyama T, Takenaka M, et al. Schwannoma of the soft palate: Report of a case. Jpn J Oral Maxillofac Surg. 1985;31 (10):2467-2470

11 Jones JA, McWilliam LJ. Intraoral neurilemmoma (schwannoma): an unusual palatal swelling. Oral Surg Oral Med Oral Pathol 1987; 63(03):351-353

12 Hieda T, Okina T, Wakae $\mathrm{H}$, et al. A case of neurilemoma of the palate. Kurume Med J 1987;34(02):75-81 
13 Krolls SO, McGinnis JP Jr, Quon D. Multinodular versus plexiform neurilemoma of the hard palate. Report of a case. Oral Surg Oral Med Oral Pathol 1994;77(02):154-157

14 Amir R, Altman KW, Zaheer S. Neurilemmoma of the hard palate. J Oral Maxillofac Surg 2002;60(09):1069-1071

15 Rabbels J, Scheer M, Heibel H, Wickenhauser C, Kübler AC. [Neurinoma of the hard palate in an 11-year-old girl. Case report]. Mund Kiefer Gesichtschir 2005;9(06):400-403

16 López-Carriches C, Baca-Pérez-Bryan R, Montalvo-Montero S. Schwannoma located in the palate: clinical case and literature review. Med Oral Patol Oral Cir Bucal 2009;14(09):e465-e468

17 Baliga M, Uppal N, Ramanathan A. Schwannomas of the head and neck: a case series. J Maxillofac Oral Surg 2009;8(03):283-286

18 Murthy VA, Ramaswamy S, Sunita M. Schwannoma of the hard palate. Indian J Otolaryngol Head Neck Surg 2009;61(Suppl 1):52-54

19 Lollar KW, Pollak N, Liess BD, Miick R, Zitsch RP III. Schwannoma of the hard palate. Am J Otolaryngol 2010;31(02):139-140

20 Parikh NR, Desai N. Intraoral schwannoma (neurilemmoma): an unusual anterior palatal swelling-A case report. J Int Oral Health 2010;2(04):87-91

21 Isildak H, Yilmaz M, Ibrahimov M, Aslan M, Karaman E, Enver O. Schwannoma of the hard palate. J Craniofac Surg 2010;21(01): 276-278

22 Santos PP, Freitas VS, Pinto LP, Freitas RA, de Souza LB. Clinicopathologic analysis of 7 cases of oral schwannoma and review of the literature. Ann Diagn Pathol 2010;14(04):235-239

23 Chawla O, North S, Yates JM. Schwannoma presenting in the soft palate of a nine-year-old boy. Dent Update 2011;38(05):327-328

24 dos Santos JN, Silva Gurgel CA, Gonçalves Ramos EA, Pereira Júnior FB, Crusoé-Rebello IM, Campos Oliveira M. Plexiform schwannoma mimicking a salivary gland tumor: an unusual case report in pediatric patient. Int J Pediatr Otorhi. 2011;6:317-321

25 Dhupar A, Yadav S, Dhupar V. Schwannoma of the hard palate: A Rare Case. The Internet J Head Neck Surg [1937-819X]. 2010; 4: [about 4p.]. Available from: http://archive.ispub.com:80/journal/ the-internet-journal-of-head-and-neck-surgery/volume-4-number-2/schwannoma-of-the-hard-palate-a-rare-case.html

26 Handschel J, Heikaus S, Depprich R, et al. Intraoral schwannoma: review of the literature and presentation of a rare case. Cranio 2012;30(02):150-153

27 Shetty SR, Mishra C, Shetty P, Kaur A, Babu S. Palatal schwannoma in an elderly woman. Gerodontology 2012;29(02):e1133-e1135

28 Prasanna Kumar D, Meghashri K. Schwannoma of the hard palate: a case report and review of literature. J Adv Oral Research. 2012; 3:24-29

29 Kapetanakis S, Vasileiadis I, Petousis A, Fiska A, Stavrianaki A. Plexiform (multinodular) schwannoma of soft palate. Report of a case. Folia Med (Plovdiv) 2012;54(03):62-64

30 Rahpeyma A, Jafarian AH, Khajeh Ahmadi S, Sarabadani J. A schwannoma of the soft palate in a child: histological and immunohistochemical features and surgical method. Iran J Otorhinolaryngol 2012;24(67):95-99

31 Venkatachala S, Krishnakumar R, Rubby SA. Soft palate schwannoma. Indian J Surg 2013;75(Suppl 1):319-321

32 Gainza-Cirauqui ML, Eguía-Del Valle A, Martínez-Conde R, CocaMeneses JC, Aguirre-Urizar JM. Ancient Schwannoma of the hard palate. An uncommon case report and review. J Clin Exp Dent 2013;5(01):e62-e65

33 Chikhale NP, Mishra A, Patel RD, Chaturvedi UP, Jayalakshmi V, Cherian S. Neurilemmoma of the hard palate: report of a case and review of literature. Int J Head Neck Surg 2013;4:123-125

34 Moradzadeh Khiavi M, Taghavi Zenouz A, Mesgarzadeh AH, Sabetmehr O, Mahmoudi SM, Kouhsoltani M. Schwannoma in the midline of hard palate: a case report and review of literature. J Dent Res Dent Clin Dent Prospect 2014;8(02):114-117

35 Aboh IV, Chisci G, Cascino F, et al. Giant palatal schwannoma. J Craniofac Surg 2014;25(05):e418e20
36 Parhar S, Singh HP, Nayyar A, Manchanda AS. Intra-oral schwannoma- a case report. J Clin Diagn Res 2014;8(03):264-265

37 Sahoo PK, Mandal PK, Ghosh S. Schwannoma of the hard palate. Natl J Maxillofac Surg 2014;5(01):39-41

38 Kudoh M, Harada H, Matsumoto K, Sato Y, Omura K, Ishii Y. Massive Neurilemoma of the Hard Plate in Which Preoperative Diagnosis Was Difficult. Case Rep Surg 2015;2015:638025

39 Meundi MA, Anekar J, A C R, Patil US, Mustafa SM. Intraosseous schwannoma of the maxilla mimicking a periapical lesion: a diagnostic challenge. J Clin Diagn Res 2015;9(03):ZD01-ZD04

40 Tibbetts KM, Cheng J. Pediatric palate schwannoma. Int J Pediatr Otorhinolaryngol Extra 2014;9:122-124

41 Yaga US, Mengji AK, Besta R, Gollamudi N. Rare case of palatal schwannoma: Case report and review of literature. J Indian Acad Oral Med Radiol. 2015;27:484-487

42 Karataş M, Sarıkaya Y, Doğan S, Erdoğdu İH, Bulut HT, Yücetaş SC. A Slow Growing Mass of Hard Palate: Schwannoma-Case Report. J Clin Med Case Reports. 2015;2(01):1-4

43 Morgan GK, Packiaraj I, Rajan A, Rahman A. Rare case of schwannoma in anterior palate. J Indian Acad Dent Spec Res. 2015;2:30-33

44 Sicca C, Cistaro A, Quartuccio N, Sardo E, Berrone M. Correlation of MRI Pattern and Histological Features in a Schwannoma of the Soft Palate in a 13-Year-Old Girl. OMICS J Radiol 2015;4(01):177

45 Barhmi I, Tazi N, Mahdoufi R, Abada R, Rouadi S. Schwannoma palate in children: rare case. J Case Rep Stud. 2016;4:202

46 Shi LL, Justicz N, Panella NJ, Henriquez OA. Soft Palate Schwannoma: A Rare Case of an Intraoral Mass. Ann. Clin Case Rep 2016; $1: 1057$

47 Eroglu CN, Keskin Tunc S, Gunhan O. Soft Tissue Schwannomas of the Hard Palate and the Mandibular Mentum. Case Rep Dent 2017;2017:7401631

48 Poonja PA, Sattur AP, Burde KN, Hallikeri K, Anehosur VS. An unusual case of neural palatal swelling. Int J Health Sci (Qassim) 2017;11(03):71-73

49 Vera-Sirera B, Fernades-Ciacha L, Floria LM, Vera-Sempere F. Palatal ancient schwannoma: optical, immunohistochemical and ultrastructural study with literature review. Eur Arch Otorhinolaryngol 2017;274(12):4195-4202

50 Gueiros LA, Barkokebas A, Carvalho AT, Leão JC. Small palatal swelling: an uncommon presentation of intraoral schwannoma. Gen Dent 2017;65(03):58-61

51 Melo CA, Almeida-Júnior P, de Carvalho Melo AU, et al. Giant schwannoma of the hard palate: a literature review and report of an uncommon case. Gen Dent 2018;66(01):62-65

52 Khalele BA. Schwannoma of the hard palate: A case report and a systematic review of literature. Future Dental Journal 2018:1-6

53 Murakami N, Fukuya Y. Surgically excising an intraoral schwannoma of the soft palate using a buccinator flap: A case report. Int J Surg Case Rep 2018;49:17-20

54 Kawakami R, Kaneko T, Kadoya M, et al. Schwannoma in the sublingual space. Dentomaxillofac Radiol 2004;33(04):259-261

55 Leu YS, Chang KC. Extracranial head and neck schwannomas: a review of 8 years experience. Acta Otolaryngol 2002;122(04):435-437

56 Williams HK, Cannell H, Silvester K, Williams DM. Neurilemmoma of the head and neck. Br J Oral Maxillofac Surg 1993;31(01):32-35

57 Lucas RB. Pathology of tumors of the oral tissue. New York, NY: Churchill Livingstone; 1984

58 Hatziotia JC, Asprides H. Neurilemoma (schwannoma) or the oral cavity. Oral Surg Oral Med Oral Pathol 1967;24(04):510-526

59 Enzinger IM, Weiss SW. Soft Tissue Tumours. 3rd ed. St Louis, MO: Mosby; 1995:821-850

60 de Lacerda SA, Brentegani LG, Rosa AL, Vespúcio MVO, Salata LA. Intraosseous schwannoma of mandibular symphysis: case report. Braz Dent J 2006;17(03):255-258

61 Hribernik SJ, Gould AR, Alpert B, Jones JL. Well-circumscribed mass of the lateral floor of the mouth. J Oral Maxillofac Surg 1992; 50(07):741-746 
62 Erlandson RA. Peripheral nerve sheath tumors. Ultrastruct Pathol 1985;9(1-2):113-122

63 Wright BA, Jackson D. Neural tumors of the oral cavity. A review of the spectrum of benign and malignant oral tumors of the oral cavity and jaws. Oral Surg Oral Med Oral Pathol 1980;49(06): 509-522

64 Oh JE, Choi YW, Choi HY, Myung KB. A case of cellular schwannoma of the lower lip. Korean J Dermatol 2008;46:1282-1284

65 Woodruff JM, Godwin TA, Erlandson RA, Susin M, Martini N. Cellular schwannoma: a variety of schwannoma sometimes mistaken for a malignant tumor. Am J Surg Pathol 1981;5(08): 733-744

66 Nonaka D, Chiriboga L, Rubin BP. Sox10: a pan-schwannian and melanocytic marker. Am J Surg Pathol 2008;32(09):1291-1298

67 Fine SW, McClain SA, Li M. Immunohistochemical staining for calretinin is useful for differentiating schwannomas from neurofibromas. Am J Clin Pathol 2004;122(04):552-559

68 Park JY, Park H, Park NJ, Park JS, Sung HJ, Lee SS. Use of Calretinin, CD56, and CD34 for Differential Diagnosis of Schwannoma and Neurofibroma. J Pathol Transl Med 2011;45(01):30-35
69 Chrysomali E, Papanicolaou SI, Dekker NP, Regezi JA. Benign neural tumors of the oral cavity: a comparative immunohistochemical study. Oral Surg Oral Med Oral Pathol Oral Radiol Endod 1997;84(04):381-390

70 Chauvin PJ, Wysocki GP, Daley TD, Pringle GA. Palisaded encapsulated neuroma of oral mucosa. Oral Surg Oral Med Oral Pathol 1992;73(01):71-74

71 Yamazaki H, Kaneko A, Ota Y, Tsukinoki K. Schwannoma of the mental nerve: usefulness of preoperative imaging: a case report. Oral Surg Oral Med Oral Pathol Oral Radiol Endod 2004;97(01):122-126

72 Colreavy MP, Lacy PD, Hughes J, et al. Head and neck schwannomas-a 10 year review. J Laryngol Otol 2000;114(02):119-124

73 Gomez-Brouchet A, Delisle MB, Cognard C, et al. Vestibular schwannomas: correlations between magnetic resonance imaging and histopathologic appearance. Otol Neurotol 2001;22(01): 79-86

74 Ghosh BC, Ghosh L, Huvos AG, Fortner JG. Malignant schwannoma. A clinicopathologic study. Cancer 1973;31(01):184-190

75 Bansal R, Trivedi P, Patel S. Schwannoma of the tongue. Oral Oncology EXTRA. 2005;41(02):15-17 